\title{
A review of the use of telemedicine technology in caring for high-risk pregnant women
}

\author{
Type of article: conference abstract
}

\author{
Malihe GhalandarAbadi1, Haleh Ayatollahi2,* \\ 1-Department of Health Information Management, School of Health Management and \\ Information Sciences, Iran University of Medical Sciences, Tehran, Iran. \\ *hayatollahi@yahoo.com
}

\begin{abstract}
Introduction: The number of high-risk pregnancy in the country is a lot, and the evidence shows that quality of care is not high for this group of people. Due to the importance of pregnancy care and its effect on the mothers' and newborns' health and the priority of prevention over treatment, serious attention needs to be paid to this group of patients in this field, like other fields of medicine, telemedicine has been used as a tool to improve the quality of care.

Aim: This study aimed to review the applications of telemedicine technology in the care of highrisk pregnant women.

Method: The was a review study which was completed by searching the databases, such as PubMed, Science Direct, Scopus, Web of knowledge and by using keywords like "mhealth", "telehealth", "high-risk pregnancy", telemedicine".

Results: The results showed that in addition to describing different telemedicine technologies in pregnancy care, advantages and disadvantages of using these technologies were discussed in a number of papers. The most important benefits of using telemedicine in high-risk pregnancy included patient satisfaction, quality of care improvement, cost reduction and fast communication between clinical staff. The main challenges of using these technologies included ethical, technical and financial challenges.

Conclusion: Reducing health care costs and improving health care delivery to the pregnant women are among the leading health policies in all countries. Despite numerous challenges in deploying telemedicine technologies, it seems that using this technology is highly acceptable among users and the advantages of using this technology can help to accelerate delivery of health care to the high-risk pregnant women.
\end{abstract}

Keywords: Pregnancy, Telemedicine, High-risk pregnancy, Homecare

\section{Declaration of conflicts}

This abstract is selected from the First International Congress of Diseases and Health Outcomes Registry and First National Congress of Medical Informatics, 14-17 February 2017, Mashhad, Iran

\section{Authors' biography}

No biography.

\section{References}

No references. 